\title{
Vorwort und Einleitung
}

Oft genug stehen Sozialforscherinnen und Sozialforscher vor scheinbar fast unlösbaren Problemen, wenn sie einen Zugang zu sozialen Lebenswelten suchen. Die empirische Sozialforschung insgesamt und die qualitative Sozialforschung im Besonderen sind daher gut beraten, neue Wege für die Forschung nicht nur zu suchen, sondern auch zu beschreiten. Dies ist aber immer auch mit dem Risiko verbunden, auf Holzwege, in Sackgassen oder auf Abwege zu geraten. So erzeugen sichtbar neue Optionen genauso häufig Euphorie wie Verunsicherung.

Für kaum einen Bereich dürfte dies so zutreffen wie für Online-Medien. Diese sind spätestens mit dem „Web 2.0“ immer wieder für die Forschung propagiert worden, ohne dass dies zu einem tatsächlichen Durchbruch der Online-Forschung geführt hätte zu groß scheinen dann doch oft die Hürden, will man nicht wieder hinter einmal erreichte Qualitätsstandards zurückfallen. Dennoch ist ein allmähliches Wachstum der Online-Forschung zu beobachten: zuerst als neuer und überaus facetten- und materialreicher Gegenstand, dann aber auch immer mehr als Ort und Medium der Datengewinnung.

Die vorliegende Arbeit befasst sich nun mit einem Bereich der Online-Forschung, der auch heute noch nur wenig und sehr zaghaft genutzt wird, nämlich mit sogenannten Forumsdiskussionen. Forumsdiskussionen, zumindest wird dieser Begriff hier so verwendet, sind online in Internet- oder Webforen (auch Bulletin Boards oder Message Boards) durchgeführte Gruppendiskussionen. Sie unterscheiden sich in ihren Merkmalen und Bedingungen nicht nur von „,normalen“ (face to face durchgeführten) Gruppendiskussionen, sondern auch von anderen mediatisierten Formen wie Chats oder Videokonferenzen.

Im Mittelpunkt steht im Folgenden die Frage, ob, wie und für welche Aufgaben Forumsdiskussionen in der qualitativen Sozialforschung sinnvoll zur Datengewinnung eingesetzt werden können. Dazu werden neben methodologischen Überlegungen vor allem Ergebnisse aus dem Forschungsprojekt „Forumsdiskussionen im Internet als qualitatives Forschungsinstrument“ vorgestellt. In diesem methodenexperimentell ausgerichteten Forschungsprojekt, das von 2015 bis 2017 von der Deutschen Forschungsgemeinschaft gefördert wurde (UL 186/7-1; SCHI 1184/3-1), wurden unterschiedlich gestaltete Forumsdiskussionen systematisch beobachtet und verglichen.

Die Durchführung dieses Forschungsvorhabens wäre ohne die Unterstützung vieler Personen und Institutionen nicht möglich gewesen. Ihnen allen soll an dieser Stelle gedankt werden. $\mathrm{Zu}$ allererst sind hier die vielen Studenten und Studentinnen unserer Universität zu nennen, die sich mehr oder weniger aktiv an den einzelnen Forumsdiskussionen als Diskutanten beteiligt haben. Darüber hinaus ist den Dekanen aller Fakultäten zu danken, die uns ihre E-Mail-Verteiler für die Gewinnung von Teilnehmer/-innen zu Verfügung gestellt haben. Gleiches gilt für die vielen Kolleginnen und Kollegen, die wir für „Werbezwecke“ in ihren Lehrveranstaltungen aufsuchen durften. 
Besonderer Dank gilt schließlich unseren ehemaligen Projektmitarbeiter/-innen und Wissenschaftlichen Hilfskräften - namentlich sind hier Sina Schadow, Christian Lukassen und Christina Schröder zu erwähnen -, ohne die die Durchführung eines solch aufwändigen Projektes undenkbar gewesen wäre. Insbesondere Christian Lukassen sei an dieser Stelle noch einmal herzlich für seine unermüdliche und unverzichtbare Unterstützung gedankt, die weit über das vertraglich gesicherte Maß hinausging.

Essen, Juli 2019

Carsten G. Ullrich

Daniela Schiek 